\title{
The Effect of Body Mass Index on Outcome after Endovascular Treatment in Acute Ischemic Stroke Patients: A Post Hoc Analysis of the MR CLEAN Trial
}

\author{
France Anne Victoire Pirson ${ }^{a}$ Wouter H. Hinsenveld ${ }^{a}$ \\ Julie Staals ${ }^{a}$ Bianca T.A. de Greef ${ }^{a}$ b Wim H. van Zwam ${ }^{c}$ \\ Diederik W.J. Dippel $^{d}$ Jan Albert Vos ${ }^{e}$ Wouter J. Schonewille \\ Robert J. van Oostenbrugge ${ }^{a}$ on behalf of MR CLEAN investigators

\begin{abstract}
${ }^{a}$ Department of Neurology, Maastricht University Medical Center, Maastricht, The Netherlands; ${ }^{b}$ Department of Clinical Epidemiology and Medical Technology Assessment, Maastricht University Medical Center, Maastricht, The Netherlands; ${ }^{C}$ Department of Radiology, Maastricht University Medical Center, Maastricht, The Netherlands; ${ }^{\mathrm{d}}$ Department of Neurology, Erasmus MC University Medical Center, Rotterdam, The Netherlands; ${ }^{\mathrm{e}}$ Department of Radiology, Sint Antonius Hospital, Nieuwegein, The Netherlands; ${ }^{\mathrm{f}}$ Department of Neurology, Sint Antonius Hospital, Nieuwegein, The Netherlands
\end{abstract}

\section{Keywords}

Body mass index · Obesity paradox · Endovascular treatment · Acute ischemic stroke · Functional outcome

\begin{abstract}
Background: Though obesity is a well-known risk factor for vascular disease, the impact of obesity on stroke outcome has been disputed. Several studies have shown that obesity is associated with better functional outcome after stroke. Whether obesity influences the benefit of endovascular treatment (EVT) in stroke patients is unknown. We evaluated the association between body mass index (BMI) and outcome in acute ischemic stroke patients with large vessel occlusion (LVO), and assessed whether BMI affects the benefit of EVT. Methods: This is a post hoc analysis of the Multicenter Randomized Clinical Trial of Endovascular Treatment for Acute Ischemic Stroke in the Netherlands trial (ISRCTN10888758). BMI was used as a continuous and cate-
\end{abstract}

\begin{tabular}{ll}
\hline KARGER & $\begin{array}{l}\text { (c) } 2019 \text { The Author(s) } \\
\text { Published by S. Karger AG, Basel }\end{array}$ \\
E-Mail karger@karger.com & This article is licensed under the Creative Commons Attribution- \\
www.karger.com/ced & $\begin{array}{l}\text { NonCommercial-NoDerivatives 4.0 International License (CC BY- } \\
\text { NC-ND) (http://www.karger.com/Services/OpenAccessLicense). } \\
\text { Usage and distribution for commercial purposes as well as any dis- } \\
\text { tribution of modified material requires written permission. }\end{array}$
\end{tabular}

gorical variable, distinguishing underweight and normal weight (BMI <25), overweight (BMI 25-30), and obesity (BMI $\geq 30$ ). We used multivariable ordinal logistic regression analysis to estimate the association of BMI with functional outcome (shift analysis), assessed with modified Rankin Scale (mRs) at 90 days. The impact of BMI on EVT effect was tested by the use of a multiplicative interaction term. Results: Of 366 patients, 160 (44\%) were underweight or normal weight, 145 (40\%) overweight, and 61 (17\%) were obese. In multivariable analysis with BMI as a continuous variable, we found a shift toward better functional outcome with higher BMI (mRS adjusted common OR 1.04; 95\% Cl 1.0-1.09), and mortality was inversely related to $\mathrm{BMI}$ (aOR $0.92 ; 95 \% \mathrm{Cl} 0.85-0.99$ ). Safety analysis showed that higher BMI was associated with lower risk of stroke progression (aOR 0.92, 95\% Cl 0.87-0.99). Additional analysis showed no interaction between BMI and EVT effect on functional outcome, mortality, and other safety outcomes. Conclusion: Our study confirms the effect of obesity on outcome in acute ischemic stroke patients with LVO, 
meaning better functional outcome, lower mortality, and lower risk of stroke progression for patients with higher BMI. As we found no interaction between BMI and EVT effect, all $\mathrm{BMI}$ classes may expect the same benefit from EVT.

(C) 2019 The Author(s)

Published by S. Karger AG, Basel

\section{Introduction}

Obesity and overweight are well-known cardiovascular risk factors and associated with an increased risk of stroke. Each unit increase of body mass index (BMI) is independently associated with $6 \%$ increase in the relative risk of stroke [1]. However, the impact of obesity on functional outcome after stroke remains controversial. Several studies have reported that obesity is independently associated with a lower mortality rate after stroke [2, 3], while other studies could not confirm this $[4,5]$.

By comparison, a better outcome for obese patients has been described in a variety of acute and chronic vascular diseases. As such, lower mortality rates and better recovery have been found in obese patients with heart failure, myocardial infarction, and chronic kidney disease $[6,7]$. This contradiction, in which obesity increases the chance of a vascular disease, yet also contributes to better recovery afterward, is known as the obesity paradox. Its underlying mechanism is yet to be explained, and therefore remains a matter of ongoing debate.

Data on the impact of obesity on stroke treatment outcomes are scarce. Most observational studies on functional outcome after intravenous thrombolysis for stroke in obese patients do not suggest an obesity paradox [8-10]. However, the obesity paradox has been confirmed in the outcome after carotid endarterectomy [11]. The impact of excess body weight on functional outcome and mortality after endovascular treatment (EVT) for acute stroke has not yet been reported.

Now that the use of EVT for large vessel occlusions (LVO) is growing and the proportion of obese patients is increasing, we feel that exploration of the obesity paradox in this specific population is relevant. We hypothesize that increased BMI may be associated with a better outcome in EVT-treated stroke patients. In this Multicenter Randomized Clinical Trial of Endovascular Treatment for Acute Ischemic Stroke in the Netherlands (MR CLEAN) post hoc analysis, we (1) investigate the relation between BMI at hospital admission and functional outcome and mortality in stroke patients and (2) assess whether BMI affects the benefit and safety of EVT in acute stroke patients.

Obesity Paradox in Acute Ischemic Stroke Patients

\section{Methods}

\section{Study Design and Patients}

In this post hoc analysis, we used data from the MR CLEAN trial [12]. The study methods and patient eligibility criteria have been reported previously [13]. Source data will not be made available because of regulatory issues on patient privacy, but detailed analytic methods and study materials, including log files of statistical analyses, will be made available to other researchers on request to the first author. MR CLEAN is a randomized, open label clinical trial of EVT versus no EVT along with usual care in patients from 16 centers in the Netherlands from 2010 until 2014. All patients were intended to be treated for a proximal arterial occlusion in the anterior circulation, within $6 \mathrm{~h}$ of symptom onset. In total, 500 patients were included in the trial, with 233 assigned to the intervention arm and 267 assigned to the control arm. The usual medical care management was performed according to national guidelines, and could include treatment with intravenous thrombolysis within the first $4.5 \mathrm{~h}$ after symptom onset.

All patients or their legal representative provided written informed consent before randomization. The study protocol was approved by a central medical Ethics Committee and the research board of each participating center.

\section{Body Mass Index}

Risk factors as well as weight and height were recorded as baseline parameters. Body weight was measured by nurses of the Emergency department or Stroke Unit or obtained verbally from the patient or their caregiver. If neither was possible, body weight and height were estimated by the nurse or anesthetic team. We calculated BMI as the ratio of body weight and squared height, given as $\mathrm{kg} / \mathrm{m}^{2}$. The patients were categorized as defined by the World Health Organization as underweight (BMI $<18.5 \mathrm{~kg} / \mathrm{m}^{2}$ ), normal weight (BMI 18.5 to $<25 \mathrm{~kg} / \mathrm{m}^{2}$ ), overweight (BMI 25 to $<30 \mathrm{~kg}$ / $\mathrm{m}^{2}$ ), obesity (BMI 30 to $<35 \mathrm{~kg} / \mathrm{m}^{2}$ ), and morbid obesity (BMI $\geq 35$ $\left.\mathrm{kg} / \mathrm{m}^{2}\right)$.

\section{Outcome and Safety Measures}

The primary outcome measure was the modified Ranking Scale (mRS) at 90 days, which is a 7 -point scale ranging from 0 (no symptoms) to 6 (death) [14]. An mRS score of 6 means a patient has died during the 90 days of follow-up. A score of 2 points or less indicates functional independence. Secondary clinical outcome measures included neurological assessment with the National Institute of Health Stroke Scale (NIHSS) at $24 \mathrm{~h}$ [15] and mortality rate by 90 days (mRS score 6 at 90 days). Secondary radiologic outcome measures included arterial recanalization on CTA or magnetic resonance angiogram at $24 \mathrm{~h}$, and final infarct volume on non-contrast CT at 5-7 days. All neuroimaging studies were evaluated by 2 neuroradiologists who were blinded for the treatmentgroup assignments.

We also tested safety parameters, which included hemorrhagic complications, progression of ischemic stroke, and pneumonia. Symptomatic intracranial hemorrhage ( $\mathrm{sICH})$ was defined as neurological deterioration of 4 or more points on the NIHSS and neuroimaging confirmed intracranial hemorrhage. The European cooperative acute stroke study (ECASS I) was used to classify intracranial hemorrhage [16]. Progression of ischemic stroke was defined as neurological deterioration with an increase of 2 or more 
points on the NIHSS and follow-up cerebral imaging compatible with the diagnosis of ischemia (in the same territory) and no other underlying cause for neurological deterioration.

\section{Statistical Analysis}

Baseline characteristics were described using standard statistics. Patients for whom we could not obtain BMI were excluded from primary assessment. For univariate analysis with unimputed data, we used categorized BMI, and for multivariable analysis, we used BMI as a continuous variable. For the relation between BMI and functional outcome, we used multivariable ordinal logistic regression analysis to estimate the adjusted common odds ratio for a shift in direction toward a better functional outcome on the mRS. Clinical and safety outcomes were adjusted for potential imbalances in pre-specified prognostic variables adapted from the original MR CLEAN trial protocol and from baseline differences: age, sex, NIHSS score at baseline, time from onset to randomization, hypertension, pre-stroke mRS score, and EVT. These variables were added to the model using a forward stepwise approach. For the interaction between BMI and EVT effect, we entered a multiplicative interaction term into the model. All descriptive analyses include patients with complete data, while all regression models include all the patients with imputed data. STATA (version 14.1) was used for all statistical analyses.

\section{Results}

\section{Patient Characteristics}

Out of 500 patients in the trial, 134 patients were excluded because of unrecorded height and/or weight leaving 366 patients for this post hoc analysis. Because few patients were categorized as "underweight $(n=5)$ " or "morbidly obese $(n=15)$," we decided to add them to the subgroups "normal weight" and "obesity," respectively. We report the comparison of patients with available BMI assessment vs. those without available BMI in online supplementary Table 1 (for all online suppl. material, see www.karger.com/doi/10.1159/000504744; Online Recourse 1). The patients for whom BMI was unavailable more often had diabetes (19 vs. $11 \%)$ and had more risk of intracranial hemorrhage (11 vs. $5 \%$ ). Baseline characteristics of the remaining 366 patients are shown in Table 1. Of these, 160 patients (44\%) were underweight or normal weight, 145 patients (40\%) were overweight, and 61 patients (17\%) were obese or morbidly obese. The presence of hypertension and the use of antiplatelet drugs were more common in the higher BMI class $(p=0.01)$. Slightly lesser number of patients were treated with EVT in the $\mathrm{BMI}<25$ groups (34\%) compared to the overweight and obese group (43\%). In the EVT group, the duration of procedure and use of general anesthesia were highest for obese patients though differences were not significant.

\section{BMI and Outcome}

The distribution of 90 day mRS scores is provided by trichotomized BMI subgroup and treatment allocation in Figure 1. In this univariate analysis, we noticed no particular shift on the mRS score between the BMI subgroups. However, for both treatment allocations, the mortality rate (mRS score 6 ) was proportionally lower in the higher BMI subgroups - respectively, 28, 19, 15\% ( $p=$ 0.36 ) for EVT-treated patients and 24, 24, 9\% for patients treated without $\operatorname{EVT}(p=0.13)$.

In multivariable analysis with BMI as a continuous variable, there was a shift toward better functional outcome (mRS at 90 days) with every point increase in BMI, adjusted common OR 1.04 (95\% CI 1.00-0.09, $p=$ 0.048; Table 2). Secondary endpoint analysis showed that the risk of mortality declined when BMI increased (aOR 0.92, 95\% CI 0.85-0.99, $p=0.02$ ), and that higher BMI was associated with lower risk of stroke progression (aOR 0.92, 95\% CI 0.87-0.99, $p=0.03$ ). All other endpoint analyses showed no significant difference in BMI.

\section{BMI and EVT Treatment Effect}

We noted no interaction between BMI and EVT effect on mRS distribution, mortality, or stroke progression (adjusted $p$ value for interaction $=0.85,0.38,0.61$ respectively). With regard to any of the other clinical or safety outcomes, there was also no treatment - BMI interaction.

\section{Discussion}

In this post hoc analysis of the MR CLEAN trial, we assessed the relation between BMI and outcome after EVT in acute stroke patients with LVO. The obesity paradox was found in terms of better functional outcome, lower mortality, and lower occurrence of stroke progression for patients with higher BMI. Additional analysis showed no interaction between BMI and EVT treatment effect.

Our results are in line with previous studies reporting better 3-month recovery in overweight and obese stroke patients compared with normal weight counterparts [1719]. It should be noted that in the study of Kim et al. [19] the association between stroke outcome and BMI lost statistical significance after adjusting for initial stroke severity, which was not the case in our study. Because several studies in obesity report milder strokes and a higher prevalence of lacunar infarctions in obese patients, initial stroke severity was assumed to be a mediator between lev- 
Table 1. Baseline characteristics of patients with available BMI and $p$ value for difference between BMI subgroups

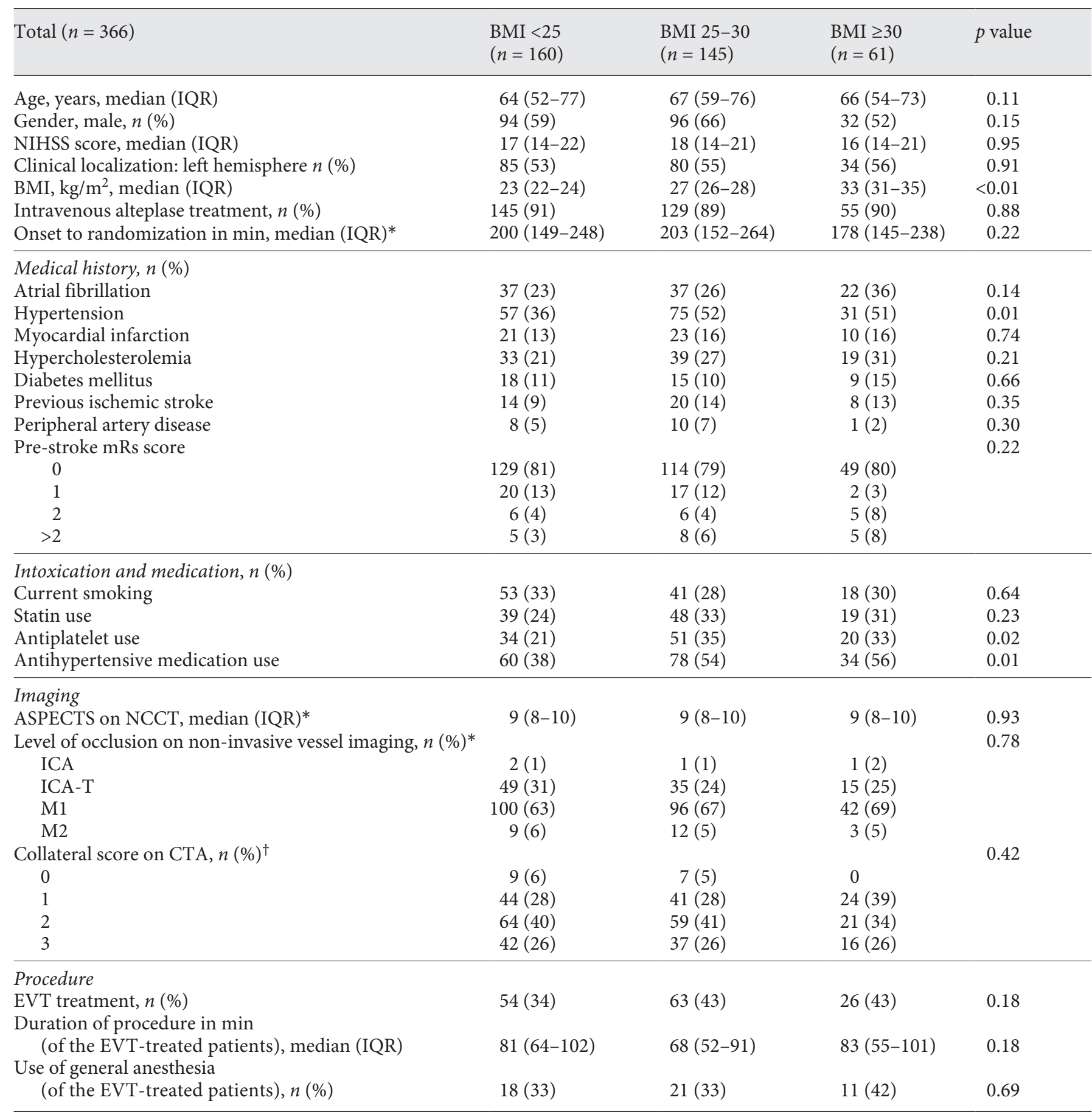

$*(n=365)$, missing in 1 patient.

$\dagger(n=364)$, missing in 2 patients because CTA was not performed or images were of insufficient quality.

BMI, body mass index; CTA, computed tomographic angiography; EVT, endovascular treatment; ICA, intracranial artery; IQR, interquartile range; NCCT, non-contrast computed tomography; NIHSS, National Institutes of Health Stroke Scale; mRS, modified Rankin Scale. 
Fig. 1. $\mathrm{mRS}$ distribution by treatment allocation per BMI subgroup. BMI, body mass index; mRs, modified Rankin Scale; EVT, endovascular treatment.

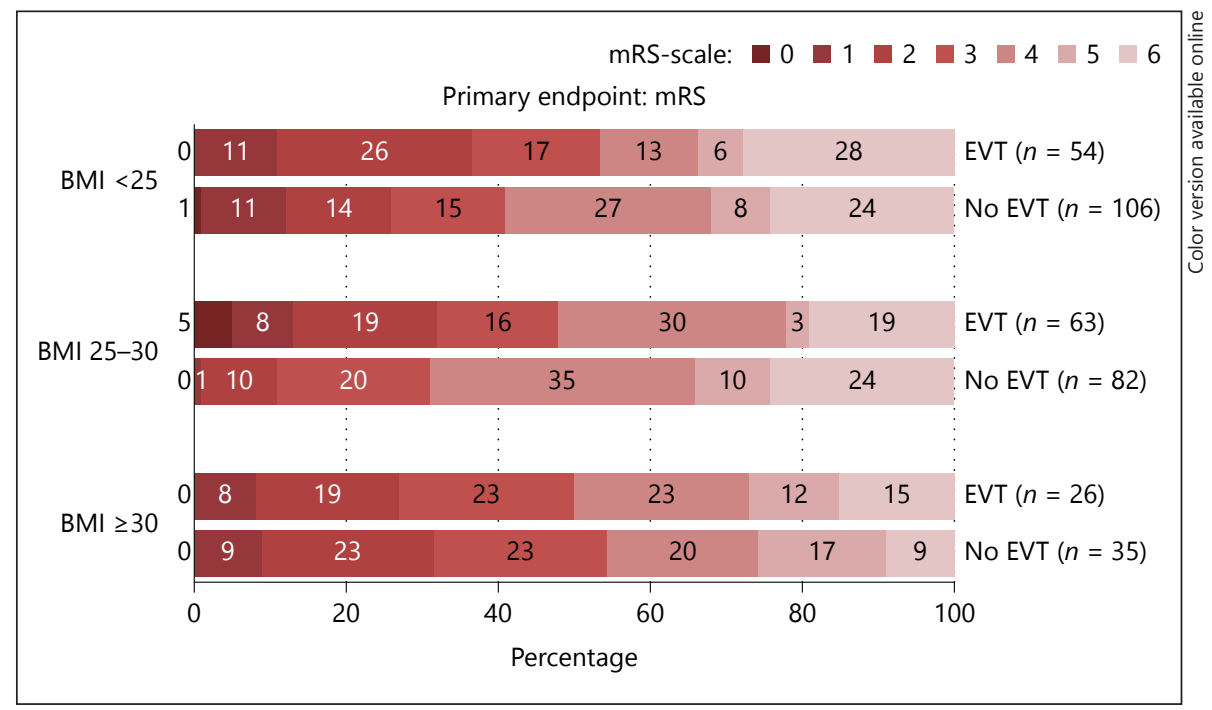

Table 2. BMI association with outcome and $p$ value for treatment interaction

\begin{tabular}{|c|c|c|c|c|}
\hline Outcome variable & $\begin{array}{l}\text { BMI } \\
\text { Unadjusted OR } \\
(95 \% \mathrm{CI})\end{array}$ & $\begin{array}{l}\text { BMI } \\
\text { Adjusted OR } \\
(95 \% \mathrm{CI})\end{array}$ & $p$ value & $\begin{array}{l}\text { BMI-treatment } \\
\text { interaction } \\
(p \text { value })\end{array}$ \\
\hline \multicolumn{5}{|l|}{ Clinical } \\
\hline mRS score at 90 days & $1.03(0.98$ to 1.07$)$ & $1.04(1.00$ to 1.09$)$ & 0.048 & 0.86 \\
\hline $\mathrm{mRS}$ score of $0-2$ at 90 days & 1.01 (0.96 to 1.06$)$ & $1.02(0.96$ to 1.08$)$ & 0.47 & 0.24 \\
\hline NIHSS score at $24 \mathrm{~h}^{*}, \beta(95 \% \mathrm{CI})$ & $0.02(-0.17$ to 0.21$)$ & $0.004(-0.16$ to 0.17$)$ & 0.96 & 0.49 \\
\hline Mortality by 90 days & $0.94(0.88$ to 1.00$)$ & $0.92(0.85$ to 0.99$)$ & 0.02 & 0.38 \\
\hline \multicolumn{5}{|l|}{ Radiological } \\
\hline No intracranial occlusion on $24 \mathrm{~h} \mathrm{CTA}^{\dagger}$ & $0.98(0.93$ to 1.03$)$ & $0.98(0.92$ to 1.03$)$ & 0.39 & 0.70 \\
\hline Final infarct volume on $\mathrm{NCCT}^{\ddagger}, \beta(95 \% \mathrm{CI})$ & $0.77(-2.94$ to 1.39$)$ & $-1.10($ to 3.14 to 0.94$)$ & 0.29 & 0.32 \\
\hline \multicolumn{5}{|l|}{ Safety outcomes } \\
\hline sICH & $1.03(0.94$ to 1.13$)$ & $1.04(0.93$ to 1.16$)$ & 0.52 & 0.78 \\
\hline Progression of stroke & $0.93(0.87$ to 0.99$)$ & $0.93(0.87$ to 0.99$)$ & 0.03 & 0.61 \\
\hline Pneumonia & $0.98(0.92$ to 1.05$)$ & $0.99(0.92$ to 1.06$)$ & 0.74 & 0.20 \\
\hline
\end{tabular}

${ }^{\dagger}(n=298)$, missing in 68 patients: imputed dataset.

$\ddagger(n=240)$, missing in 126 patients: imputed dataset.

$* n=358$, missing in 8 patients: imputed dataset.

$\beta$, regression coefficient, estimated with linear regression analyses.

OR for BMI association with outcome variable, estimated with logistic regression analyses. Adjustments were made for age, gender, NIHSS score at baseline, time from onset to randomization, hypertension, pre-mRS score, and EVT.

BMI, body mass index; CTA, computed tomographic angiography; IQR, interquartile range; mRS, modified Rankin Scale; NCCT, non to contrast computed tomography; NIHSS, National Institutes of Health Stroke Scale; sICH, symptomatic intracranial hemorrhage.

els of BMI and post-stroke outcome, contradicting the existence of obesity paradox $[4,20]$. However, our study concerns a distinct patient population with LVO, which is reflected in less influence of NIHSS dispersion and initial stroke severity.
Apart from better functional outcome, our study also reports an inverse relation between BMI and poststroke mortality. By comparison, Olsen et al. [2] demonstrated that post-stroke mortality was lowest in overweight and obese patients. This inverse relation was 
also found in successive studies, though most of them reported on long-term mortality (1-2 years) [4, 21]. Long-term mortality is more likely to be influenced by comorbidity other than stroke and acute treatment. Therefore, we restricted our analyses to short-term mortality (within 90 days) in order to limit the risk of confounding. Furthermore, most studies also show higher mortality rates in underweight patients $[2,3$, $22]$. Our study, however, had only 5 patients who were underweight (1.4\% of study population), which may have influenced the effect on outcome by limited BMI dispersion.

Post-stroke recovery is likely to be influenced by weight loss and muscle tissue degradation. Excess body weight may therefore have a protective effect by providing patients with metabolic reserves. From a biological point of view, the obesity paradox could be explained by potential protective effects of adipose tissue, as it blocks the release of inflammatory cytokines during catabolic state [23]. We consider this as a possible explanation for lower occurrence of stroke progression in our study. However, the exact underlying mechanism is still unclear, and we cannot exclude potential treatment bias as another cause of the obesity paradox, as it is possible that physicians and paramedics treat obese patients more aggressively due to assumed increased risk of recurrent events.

The strength of our study is the randomized trial design that enabled us to evaluate the impact of BMI on treatment effect. We note that the advantage of randomization does not apply in our comparison between BMI subgroups. Furthermore, all outcome measures have been collected prospectively according to protocol and irrespective of our present research question.

Nevertheless, there are several limitations. First, the reported weight and height often resulted from estimation, especially in an emergency setting. It is therefore possible that BMI measures were not obtained in a uniform way. Also, the diagnostic performance of BMI diminishes by increasing age, and alternative tools such as waist-to-hip ratio or waist circumference are proven to be more precise in the measurement of obesity [24, 25]. However, in clinical practice, BMI measurement is still the most pragmatic option.

Second, we note that in our study, the association between BMI and functional outcome is most likely influenced by the strong association between BMI and mortality since mortality is embedded in the mRS score.

Third limitation concerns our post hoc design with the risk of inclusion bias. In our study, a rather large

Obesity Paradox in Acute Ischemic Stroke

Patients proportion of patients were excluded from analysis because of missing BMI. Additional analyses (online suppl. Table 1) showed no difference in mortality rate between the included and excluded cohorts, though we did find a significant difference in the occurrence of sICH. Patient with missing BMI had higher occurrence of sICH compared to patients with no missing BMI. Consequently, selection bias could have affected our secondary outcome analysis while it likely has no effect on the primary and other secondary outcome measures.

In summary, in this post hoc analysis of a large randomized controlled trial, we found a positive effect of increased BMI on functional outcome and mortality in acute ischemic stroke patients with LVO, confirming the obesity paradox. Safety analysis also showed a lower risk of stroke progression in patients with higher BMI. However, there was no interaction between BMI and EVT effect, which means all the BMI classes may expect the same benefit from EVT.

\section{Disclosure Statement}

Dr. Wim H. van Zwam reports personal fees from Stryker and personal fees from Cerenovus (paid to institution). Dr. Diederik W.J. Dippel reports grants from Dutch Heart Foundation, grants from the Brain Foundation Netherlands, grants from the Netherlands Organisation for Health Research and Development, grants from Health Holland Top Sector Life Sciences and Health, grants from AngioCare BV, grants from Medtronic/Covidien/EV3, grants from MEDAC Gmbh/LAMEPRO, grants from Penumbra Inc., grants from Top Medical/Concentric, grants from Stryker, and grants from Thrombolytic Science during the conduct of the study.

\section{Funding Sources}

The MR CLEAN trial was partly funded by the Dutch Heart Foundation and by unrestricted grants from AngioCare BV, Medtronic/Covidien/EV3 ${ }^{\circledR}$, MEDAC Gmbh/LAMEPRO, Penumbra Inc., Stryker ${ }^{\circledR}$, and Top Medical/Concentric. The MR CLEAN is registered under number NTR1804 in the Dutch trial register and under ISRCTN10888758 in the ISRCTN register.

\section{Author Contributions}

F.A.V.P. performed the analyses and wrote the first draft of the manuscript. B.T.A.G and W.H.H. contributed to the design of the used statistical models. All other authors critically reviewed the manuscript for intellectual content. All authors read and approved the final version of the manuscript. 


\section{References}

1 Kurth T, Gaziano JM, Berger K, Kase CS, Rexrode KM, Cook NR, et al. Body mass index and the risk of stroke in men. Arch Intern Med. 2002 Dec;162(22):2557-62.

2 Olsen TS, Dehlendorff C, Petersen HG, Andersen KK. Body mass index and poststroke mortality. Neuroepidemiology. 2008;30(2): 93-100.

3 Doehner W, Schenkel J, Anker SD, Springer J, Audebert HJ. Overweight and obesity are associated with improved survival, functional outcome, and stroke recurrence after acute stroke or transient ischaemic attack: observations from the TEMPiS trial. Eur Heart J. 2013 Jan;34(4):268-77.

4 Ryu WS, Lee SH, Kim CK, Kim BJ, Yoon BW. Body mass index, initial neurological severity and long-term mortality in ischemic stroke. Cerebrovasc Dis. 2011;32(2):170-6.

5 Dehlendorff C, Andersen KK, Olsen TS. Body mass index and death by stroke: no obesity paradox. JAMA Neurol. 2014 Aug;71(8):978-84.

6 Fonarow GC, Srikanthan P, Costanzo MR, Cintron GB, Lopatin M; ADHERE Scientific Advisory Committee and Investigators. An obesity paradox in acute heart failure: analysis of body mass index and inhospital mortality for 108,927 patients in the Acute Decompensated Heart Failure National Registry. Am Heart J. 2007 Jan;153(1):74-81.

7 Kalantar-Zadeh K, Block G, Humphreys MH, Kopple JD. Reverse epidemiology of cardiovascular risk factors in maintenance dialysis patients. Kidney Int. 2003 Mar;63(3):793808.

8 Diedler J, Ahmed N, Glahn J, Grond M, Lorenzano $S$, Brozman $M$, et al. Is the maximum dose of $90 \mathrm{mg}$ alteplase sufficient for patients with ischemic stroke weighing $>100 \mathrm{~kg}$ ? Stroke. 2011 Jun;42(6):1615-20.

9 Sarikaya H, Arnold M, Engelter ST, Lyrer PA, Mattle HP, Michel P, et al. Outcome of intra- venous thrombolysis in stroke patients weighing over $100 \mathrm{~kg}$. Cerebrovasc Dis. 2011;32(3): 201-6.

10 Sarikaya H, Elmas F, Arnold M, Georgiadis D, Baumgartner RW. Impact of obesity on stroke outcome after intravenous thrombolysis. Stroke. 2011 Aug;42(8):2330-2.

11 Jackson RS, Black JH 3rd, Lum YW, Schneider EB, Freischlag JA, Perler BA, et al. Class I obesity is paradoxically associated with decreased risk of postoperative stroke after carotid endarterectomy. J V asc Surg. 2012 May; 55(5):1306-12.

12 Berkhemer OA, Fransen PS, Beumer D, van den Berg LA, Lingsma HF, Yoo AJ, et al.; MR CLEAN Investigators. A randomized trial of intraarterial treatment for acute ischemic stroke. N Engl J Med. 2015 Jan;372(1):11-20.

13 Fransen PS, Beumer D, Berkhemer OA, van den Berg LA, Lingsma $H$, van der Lugt A, et al.; MR CLEAN Investigators. MR CLEAN, a multicenter randomized clinical trial of endovascular treatment for acute ischemic stroke in the Netherlands: study protocol for a randomized controlled trial. Trials. 2014 Sep; 15(1):343.

14 van Swieten JC, Koudstaal PJ, Visser MC, Schouten HJ, van Gijn J. Interobserver agreement for the assessment of handicap in stroke patients. Stroke. 1988 May;19(5):604-7.

15 Brott T, Adams HP Jr, Olinger CP, Marler JR, Barsan WG, Biller J, et al. Measurements of acute cerebral infarction: a clinical examination scale. Stroke. 1989 Jul;20(7):864-70.

16 Fiorelli M, Bastianello S, von Kummer R, del Zoppo GJ, Larrue V, Lesaffre E, et al. Hemorrhagic transformation within 36 hours of a cerebral infarct: relationships with early clinical deterioration and 3-month outcome in the European Cooperative Acute Stroke Study I (ECASS I) cohort. Stroke. 1999 Nov;30(11): 2280-4.
17 Zhao L, Du W, Zhao X, Liu L, Wang C, Wang $\mathrm{Y}$, et al. Favorable functional recovery in overweight ischemic stroke survivors: findings from the China National Stroke Registry. J Stroke Cerebrovasc Dis. 2014 Mar;23(3): e201-6.

18 Burke DT, Al-Adawi S, Bell RB, Easley K, Chen S, Burke DP. Effect of body mass index on stroke rehabilitation. Arch Phys Med Rehabil. 2014 Jun;95(6):1055-9.

19 Kim Y, Kim CK, Jung S, Yoon BW, Lee SH. Obesity-stroke paradox and initial neurological severity. J Neurol Neurosurg Psychiatry. 2015 Jul;86(7):743-7.

20 Ovbiagele B, Bath PM, Cotton D, Vinisko R, Diener HC. Obesity and recurrent vascular risk after a recent ischemic stroke. Stroke. 2011 Dec;42(12):3397-402.

21 Kim BJ, Lee SH, Jung KH, Yu KH, Lee BC, Roh JK; For Korean Stroke Registry investigators. Dynamics of obesity paradox after stroke, related to time from onset, age, and causes of death. Neurology. 2012 Aug;79(9): 856-63.

22 Sun W, Huang Y, Xian Y, Zhu S, Jia Z, Liu R et al. Association of body mass index with mortality and functional outcome after acute ischemic stroke. Sci Rep. 2017 May;7(1): 2507.

23 Scherbakov N, Dirnagl U, Doehner W. Body weight after stroke: lessons from the obesity paradox. Stroke. 2011 Dec;42(12):3646-50.

24 Romero-Corral A, Somers VK, Sierra-Johnson J, Thomas RJ, Collazo-Clavell ML, Korinek $\mathrm{J}$, et al. Accuracy of body mass index in diagnosing obesity in the adult general population. Int J Obes. 2008 Jun;32(6):959-66.

25 Janssen I, Katzmarzyk PT, Ross R. Body mass index is inversely related to mortality in older people after adjustment for waist circumference. J Am Geriatr Soc. 2005 Dec;53(12): 2112-8 